\title{
The reciprocal sums of even and odd terms in the Fibonacci sequence
}

\author{
Andrew YZ Wang* and Fan Zhang
}

"Correspondence:

yzwang@uestc.edu.cn

School of Mathematical Sciences, University of Electronic Science and

Technology of China, Chengdu, 611731, P.R. China

\begin{abstract}
In this paper, we investigate the reciprocal sums of even and odd terms in the Fibonacci sequence, and we obtain four interesting families of identities which give the partial finite sums of the even-indexed (resp., odd-indexed) reciprocal Fibonacci numbers and the even-indexed (resp., odd-indexed) squared reciprocal Fibonacci numbers.
\end{abstract}

MSC: $11 \mathrm{~B} 39$

Keywords: Fibonacci sequence; even and odd term; reciprocal sum

\section{Introduction}

The Fibonacci sequence is defined by the linear recurrence relation

$$
F_{n}=F_{n-1}+F_{n-2} \quad \text { for } n \geq 2 \text {, }
$$

where $F_{n}$ is called the $n$th Fibonacci number with $F_{0}=0$ and $F_{1}=1$. There exists a simple and non-obvious formula for the Fibonacci numbers,

$$
F_{n}=\frac{1}{\sqrt{5}}\left(\frac{1+\sqrt{5}}{2}\right)^{n}-\frac{1}{\sqrt{5}}\left(\frac{1-\sqrt{5}}{2}\right)^{n} .
$$

The Fibonacci sequence plays an important role in the theory and applications of mathematics, and its various properties have been investigated by many authors; see [1-5].

In recent years, there has been an increasing interest in studying the reciprocal sums of the Fibonacci numbers. For example, Elsner et al. [6-9] investigated the algebraic relations for reciprocal sums of the Fibonacci numbers. In [10], the partial infinite sums of the reciprocal Fibonacci numbers were studied by Ohtsuka and Nakamura. They established the following results, where $\lfloor\cdot\rfloor$ denotes the floor function.

Theorem 1.1 For all $n \geq 2$,

$$
\left\lfloor\left(\sum_{k=n}^{\infty} \frac{1}{F_{k}}\right)^{-1}\right\rfloor= \begin{cases}F_{n-2}, & \text { if } n \text { is even } ; \\ F_{n-2}-1, & \text { if } n \text { is odd } .\end{cases}
$$

(c) 2015 Wang and Zhang. This article is distributed under the terms of the Creative Commons Attribution 4.0 International License (http://creativecommons.org/licenses/by/4.0/), which permits unrestricted use, distribution, and reproduction in any medium, provided you give appropriate credit to the original author(s) and the source, provide a link to the Creative Commons license, and indicate if changes were made. 
Theorem 1.2 For each $n \geq 1$,

$$
\left\lfloor\left(\sum_{k=n}^{\infty} \frac{1}{F_{k}^{2}}\right)^{-1}\right\rfloor= \begin{cases}F_{n} F_{n-1}-1, & \text { if } n \text { is even } \\ F_{n} F_{n-1}, & \text { if } n \text { is odd } .\end{cases}
$$

Wu and Zhang [11, 12] generalized these identities to the Fibonacci polynomials and Lucas polynomials, and they considered the subseries of infinite sums derived from the reciprocals of the Fibonacci polynomials and Lucas polynomials.

Recently, Wu and Wang [13] studied the partial finite sum of the reciprocal Fibonacci numbers and deduced the following main result.

Theorem 1.3 For all $n \geq 4$,

$$
\left\lfloor\left(\sum_{k=n}^{2 n} \frac{1}{F_{k}}\right)^{-1}\right\rfloor=F_{n-2} .
$$

Inspired by Wu and Wang's work, Wang and Wen [14] strengthened Theorem 1.1 and 1.2 to the finite sum case.

Theorem 1.4 If $m \geq 3$ and $n \geq 2$, then

$$
\left\lfloor\left(\sum_{k=n}^{m n} \frac{1}{F_{k}}\right)^{-1}\right\rfloor= \begin{cases}F_{n-2}, & \text { if } n \text { is even; } \\ F_{n-2}-1, & \text { if } n \text { is odd. }\end{cases}
$$

Theorem 1.5 For all $m \geq 2$ and $n \geq 1$, we have

$$
\left\lfloor\left(\sum_{k=n}^{m n} \frac{1}{F_{k}^{2}}\right)^{-1}\right\rfloor= \begin{cases}F_{n} F_{n-1}-1, & \text { if } n \text { is even } ; \\ F_{n} F_{n-1}, & \text { if } n \text { is odd } .\end{cases}
$$

Applying elementary methods, we investigate the partial finite sums of the even-indexed and odd-indexed reciprocal Fibonacci numbers in this paper, and obtain four interesting families of identities. In Section 2, we consider the reciprocal sums of even and odd terms in the Fibonacci sequence. In Section 3, we present the finite sums of the even-indexed and odd-indexed squared reciprocal Fibonacci numbers.

\section{Main results I: the reciprocal sums}

We first present several well-known results on Fibonacci numbers, which will be used throughout the article. The detailed proofs can be found in [5].

Lemma 2.1 Let $n \geq 1$, we have

$$
F_{n}^{2}-F_{n-1} F_{n+1}=(-1)^{n-1}
$$

and

$$
F_{a} F_{b}+F_{a+1} F_{b+1}=F_{a+b+1}
$$

if $a$ and $b$ are positive integers. 
As a consequence of (2.2), we have the following result.

Corollary 2.2 If $n \geq 1$, then

$$
\begin{aligned}
& F_{2 n}=F_{n+1}^{2}-F_{n-1}^{2}, \\
& F_{2 n+1}=F_{n-1} F_{n+1}+F_{n} F_{n+2}, \\
& F_{2 n+1}=F_{n+1} F_{n+2}-F_{n-1} F_{n} .
\end{aligned}
$$

The following is an interesting identity concerning the Fibonacci numbers.

Lemma 2.3 Assume that $a$ and $b$ are two integers with $a \geq b \geq 0$. If $n>a$, then

$$
F_{n+a} F_{n-a-1}-F_{n+b} F_{n-b-1}=(-1)^{n-a} F_{a+b+1} F_{a-b}
$$

Proof We proceed by induction on $n$. It is clearly true for $n=a+1$. Assuming the result holds for any integer $n>a$, we show that the same is true for $n+1$.

Applying (2.2) repeatedly and by the induction hypothesis, we get

$$
\begin{aligned}
F_{(n+1)+a} F_{(n+1)-a-1}-F_{(n+1)+b} F_{(n+1)-b-1}= & \left(F_{n+1+a} F_{n-a}+F_{n+a} F_{n-a-1}\right) \\
& -F_{n+1+b} F_{n-b}-F_{n+a} F_{n-a-1} \\
= & F_{2 n}-F_{n+1+b} F_{n-b}-F_{n+a} F_{n-a-1} \\
= & F_{n+b} F_{n-b-1}-F_{n+a} F_{n-a-1} \\
= & -\left(F_{n+a} F_{n-a-1}-F_{n+b} F_{n-b-1}\right) \\
= & (-1)^{n+1-a} F_{a+b+1} F_{a-b},
\end{aligned}
$$

which completes the induction on $n$.

Remark Recently, Akyiğit et al. [15, 16] defined the split Fibonacci quaternion, the split Lucas quaternion and the split generalized Fibonacci quaternion, and they obtained some similar identities to those above for these quaternions.

Before presenting our main results, we establish an inequality.

Proposition 2.4 If $n \geq 3$, then

$$
\frac{1}{F_{4 n+1}}>\sum_{k=n}^{2 n} \frac{1}{F_{2 k-1} F_{2 k} F_{2 k+1}} .
$$

Proof A direct calculation shows that it is true for $n=3$. Thus, we assume that $n \geq 4$ in the rest of the proof.

Setting $a=2$ and $b=0$, and replacing $n$ by $2 n$ in (2.6) yields

$$
F_{2 n+2} F_{2 n-3}=F_{2 n} F_{2 n-1}+2 \text {. }
$$


From (2.5), we know that

$$
F_{4 n+1}=F_{2 n+1} F_{2 n+2}-F_{2 n-1} F_{2 n} .
$$

Applying (2.8), (2.9), and the fact $F_{2 n-3} \geq 2$ and $F_{2 n-1} F_{2 n}>F_{2 n+1}$ if $n \geq 3$, we obtain

$$
\begin{aligned}
F_{2 n-3} F_{4 n+1} & =F_{2 n-3}\left(F_{2 n+1} F_{2 n+2}-F_{2 n-1} F_{2 n}\right) \\
& =F_{2 n-3} F_{2 n+1} F_{2 n+2}-F_{2 n-3} F_{2 n-1} F_{2 n} \\
& =\left(F_{2 n-1} F_{2 n}+2\right) F_{2 n+1}-F_{2 n-3} F_{2 n-1} F_{2 n} \\
& =F_{2 n-1} F_{2 n} F_{2 n+1}+2 F_{2 n+1}-F_{2 n-3} F_{2 n-1} F_{2 n} \\
& =F_{2 n-1} F_{2 n} F_{2 n+1}-\left(F_{2 n-3} F_{2 n-1} F_{2 n}-2 F_{2 n+1}\right) \\
& <F_{2 n-1} F_{2 n} F_{2 n+1},
\end{aligned}
$$

which is equivalent to

$$
\frac{F_{4 n+1}}{F_{2 n-1} F_{2 n} F_{2 n+1}}<\frac{1}{F_{2 n-3}} .
$$

Now we have

$$
\begin{aligned}
\frac{1}{F_{4 n+1}}-\sum_{k=n}^{2 n} \frac{1}{F_{2 k-1} F_{2 k} F_{2 k+1}} & =\frac{1}{F_{4 n+1}}-\frac{1}{F_{4 n+1}} \sum_{k=n}^{2 n} \frac{F_{4 n+1}}{F_{2 k-1} F_{2 k} F_{2 k+1}} \\
& >\frac{1}{F_{4 n+1}}-\frac{1}{F_{4 n+1}} \sum_{k=n}^{2 n} \frac{1}{F_{2 n-3}} \\
& =\frac{1}{F_{4 n+1}}\left(\frac{F_{2 n-3}-n-1}{F_{2 n-3}}\right) .
\end{aligned}
$$

It is not hard to see that for $n \geq 4, F_{2 n-3} \geq n+1$, which completes the proof.

Now we introduce our main results on the reciprocal sums of Fibonacci numbers.

Theorem 2.5 For all $n \geq 3$, we have

$$
\left\lfloor\left(\sum_{k=n}^{2 n} \frac{1}{F_{2 k}}\right)^{-1}\right\rfloor=F_{2 n-1} .
$$

Proof By elementary manipulations and (2.1), we derive that, for $k \geq 1$,

$$
\begin{aligned}
\frac{1}{F_{2 k-1}+1}-\frac{1}{F_{2 k}}-\frac{1}{F_{2 k+1}+1}= & \frac{F_{2 k}\left(F_{2 k+1}-F_{2 k-1}\right)-\left(F_{2 k-1}+1\right)\left(F_{2 k+1}+1\right)}{F_{2 k}\left(F_{2 k-1}+1\right)\left(F_{2 k+1}+1\right)} \\
& =\frac{F_{2 k}^{2}-F_{2 k-1} F_{2 k+1}-F_{2 k-1}-F_{2 k+1}-1}{F_{2 k}\left(F_{2 k-1}+1\right)\left(F_{2 k+1}+1\right)} \\
& =\frac{(-1)^{2 k-1}-F_{2 k-1}-F_{2 k+1}-1}{F_{2 k}\left(F_{2 k-1}+1\right)\left(F_{2 k+1}+1\right)}
\end{aligned}
$$


Hence, we have

$$
\begin{aligned}
\sum_{k=n}^{2 n} \frac{1}{F_{2 k}} & =\frac{1}{F_{2 n-1}+1}-\frac{1}{F_{4 n+1}+1}+\sum_{k=n}^{2 n}\left(\frac{1}{F_{2 k}\left(F_{2 k-1}+1\right)}+\frac{1}{F_{2 k}\left(F_{2 k+1}+1\right)}\right) \\
& >\frac{1}{F_{2 n-1}+1}-\frac{1}{F_{4 n+1}+1}+\frac{1}{F_{2 n}\left(F_{2 n-1}+1\right)} .
\end{aligned}
$$

It follows from (2.4) that

$$
F_{4 n+1}>F_{2 n} F_{2 n+2}>F_{2 n}\left(F_{2 n-1}+1\right),
$$

which implies that

$$
\sum_{k=n}^{2 n} \frac{1}{F_{2 k}}>\frac{1}{F_{2 n-1}+1}
$$

Invoking (2.1) again, we can readily deduce that

$$
\frac{1}{F_{2 k-1}}-\frac{1}{F_{2 k}}-\frac{1}{F_{2 k+1}}=\frac{-1}{F_{2 k-1} F_{2 k} F_{2 k+1}}
$$

from which we obtain

$$
\sum_{k=n}^{2 n} \frac{1}{F_{2 k}}=\frac{1}{F_{2 n-1}}-\frac{1}{F_{4 n+1}}+\sum_{k=n}^{2 n} \frac{1}{F_{2 k-1} F_{2 k} F_{2 k+1}} .
$$

Because of (2.7), we get, if $n \geq 3$,

$$
\sum_{k=n}^{2 n} \frac{1}{F_{2 k}}<\frac{1}{F_{2 n-1}}
$$

Combining (2.11) and (2.13), we have

$$
\frac{1}{F_{2 n-1}+1}<\sum_{k=n}^{2 n} \frac{1}{F_{2 k}}<\frac{1}{F_{2 n-1}}
$$

which yields the desired identity

Theorem 2.6 If $m \geq 3$ and $n \geq 1$, we have

$$
\left\lfloor\left(\sum_{k=n}^{m n} \frac{1}{F_{2 k}}\right)^{-1}\right\rfloor=F_{2 n-1}-1 .
$$

Proof It is obviously true for $n=1$. Now we assume that $n \geq 2$.

By some calculations and (2.1), we obtain, for $k \geq 2$,

$$
\frac{1}{F_{2 k-1}-1}-\frac{1}{F_{2 k}}-\frac{1}{F_{2 k+1}-1}=\frac{F_{2 k-1}+F_{2 k+1}-2}{\left(F_{2 k-1}-1\right) F_{2 k}\left(F_{2 k+1}-1\right)}>0,
$$


from which we have

$$
\sum_{k=n}^{m n} \frac{1}{F_{2 k}}<\frac{1}{F_{2 n-1}-1}-\frac{1}{F_{2 m n+1}-1}<\frac{1}{F_{2 n-1}-1}
$$

On the other hand, it follows from (2.12) that

$$
\begin{aligned}
\sum_{k=n}^{m n} \frac{1}{F_{2 k}} & =\frac{1}{F_{2 n-1}}-\frac{1}{F_{2 m n+1}}+\sum_{k=n}^{m n} \frac{1}{F_{2 k-1} F_{2 k} F_{2 k+1}} \\
& >\frac{1}{F_{2 n-1}}+\frac{1}{F_{2 n-1} F_{2 n} F_{2 n+1}}-\frac{1}{F_{2 m n+1}} .
\end{aligned}
$$

We claim that if $n \geq 1$ and $m \geq 3$,

$$
F_{2 n-1} F_{2 n} F_{2 n+1}<F_{2 m n+1} .
$$

Replacing $a$ by $a-1$ in (2.2), we arrive at

$$
F_{a-1} F_{b}+F_{a} F_{b+1}=F_{a+b}
$$

which implies that

$$
F_{a+b} \geq F_{a} F_{b+1} \geq F_{a} F_{b}
$$

Thus, $F_{2 n-1} F_{2 n} F_{2 n+1} \leq F_{6 n}<F_{6 n+1} \leq F_{2 m n+1}$, which means

$$
\sum_{k=n}^{m n} \frac{1}{F_{2 k}}>\frac{1}{F_{2 n-1}}
$$

Combining (2.16) and (2.18) yields

$$
\frac{1}{F_{2 n-1}}<\sum_{k=n}^{m n} \frac{1}{F_{2 k}}<\frac{1}{F_{2 n-1}-1}
$$

from which the desired result follows immediately.

Corollary 2.7 For all $n \geq 1$, we have

$$
\left\lfloor\left(\sum_{k=n}^{\infty} \frac{1}{F_{2 k}}\right)^{-1}\right\rfloor=F_{2 n-1}-1 .
$$

Proof By using (2.15) repeatedly, we have

$$
\begin{aligned}
\frac{1}{F_{2 n-1}-1} & >\frac{1}{F_{2 n}}+\frac{1}{F_{2 n+1}-1} \\
& >\frac{1}{F_{2 n}}+\frac{1}{F_{2 n+2}}+\frac{1}{F_{2 n+3}-1} \\
& >\frac{1}{F_{2 n}}+\frac{1}{F_{2 n+2}}+\frac{1}{F_{2 n+4}}+\cdots .
\end{aligned}
$$


Thus, we obtain

$$
\sum_{k=n}^{\infty} \frac{1}{F_{2 k}}<\frac{1}{F_{2 n-1}-1}
$$

Applying the same argument to (2.12) yields

$$
\sum_{k=n}^{\infty} \frac{1}{F_{2 k}}>\frac{1}{F_{2 n-1}}
$$

Hence we have

$$
\frac{1}{F_{2 n-1}}<\sum_{k=n}^{\infty} \frac{1}{F_{2 k}}<\frac{1}{F_{2 n-1}-1}
$$

which completes the proof.

Remark Identity (2.19) can be regarded as the limit of (2.14) as $m \rightarrow \infty$.

Theorem 2.8 For all $n \geq 1$ and $m \geq 2$, we have

$$
\left\lfloor\left(\sum_{k=n}^{m n} \frac{1}{F_{2 k-1}}\right)^{-1}\right\rfloor=F_{2 n-2}
$$

Proof It is clearly true for $n=1$, hence we suppose that $n \geq 2$ in the following. Invoking (2.1), we derive that for $k \geq 2$,

$$
\frac{1}{F_{2 k-2}}-\frac{1}{F_{2 k-1}}-\frac{1}{F_{2 k}}=\frac{1}{F_{2 k-2} F_{2 k-1} F_{2 k}}>0,
$$

which implies that

$$
\sum_{k=n}^{m n} \frac{1}{F_{2 k-1}}<\frac{1}{F_{2 n-2}}-\frac{1}{F_{2 m n}}<\frac{1}{F_{2 n-2}} .
$$

It follows from (2.17) that

$$
F_{4 n+1}>F_{2 n+1} F_{2 n}>\left(F_{2 n-2}+1\right) F_{2 n-1},
$$

based on which we conclude that, when $n>1$,

$$
\frac{F_{2 n}+F_{2 n-2}}{\left(F_{2 n-2}+1\right) F_{2 n-1}\left(F_{2 n}+1\right)}>\frac{1}{\left(F_{2 n-2}+1\right) F_{2 n-1}}>\frac{1}{F_{4 n+1}} \geq \frac{1}{F_{2 m n+1}} .
$$

Employing (2.1) again, we can readily obtain

$$
\frac{1}{F_{2 k-2}+1}-\frac{1}{F_{2 k-1}}-\frac{1}{F_{2 k}+1}=\frac{-F_{2 k}-F_{2 k-2}}{\left(F_{2 k-2}+1\right) F_{2 k-1}\left(F_{2 k}+1\right)},
$$


from which we arrive at

$$
\begin{aligned}
\sum_{k=n}^{m n} \frac{1}{F_{2 k-1}} & =\frac{1}{F_{2 n-2}+1}-\frac{1}{F_{2 m n}+1}+\sum_{k=n}^{m n} \frac{F_{2 k}+F_{2 k-2}}{\left(F_{2 k-2}+1\right) F_{2 k-1}\left(F_{2 k}+1\right)} \\
& >\frac{1}{F_{2 n-2}+1}-\frac{1}{F_{2 m n}+1}+\frac{F_{2 n}+F_{2 n-2}}{\left(F_{2 n-2}+1\right) F_{2 n-1}\left(F_{2 n}+1\right)} \\
& >\frac{1}{F_{2 n-2}+1} .
\end{aligned}
$$

Combining the above inequality with (2.21), we have

$$
\frac{1}{F_{2 n-2}+1}<\sum_{k=n}^{m n} \frac{1}{F_{2 k-1}}<\frac{1}{F_{2 n-2}}
$$

which yields the desired result.

As $m$ approaches infinity, Theorem 2.8 becomes the following.

Corollary 2.9 If $n \geq 1$, we have

$$
\left\lfloor\left(\sum_{k=n}^{\infty} \frac{1}{F_{2 k-1}}\right)^{-1}\right\rfloor=F_{2 n-2} .
$$

\section{Main results II: the reciprocal square sums}

We first introduce several preliminary results on the square of the Fibonacci numbers.

Lemma 3.1 For all $n \geq 2$, we have

$$
F_{n-1}^{2} F_{n+1}^{2}-F_{n-2}^{2} F_{n+2}^{2}=(-1)^{n} \cdot 4 \cdot F_{n}^{2} .
$$

Proof It follows from

$$
\begin{aligned}
F_{n-2} F_{n+2} & =\left(F_{n}-F_{n-1}\right)\left(F_{n}+F_{n+1}\right) \\
& =F_{n}^{2}+F_{n} F_{n+1}-F_{n-1} F_{n}-F_{n-1} F_{n+1} \\
& =2 F_{n}^{2}-F_{n-1} F_{n+1}
\end{aligned}
$$

that

$$
\begin{aligned}
F_{n-1}^{2} F_{n+1}^{2}-F_{n-2}^{2} F_{n+2}^{2} & =\left(F_{n-1} F_{n+1}+F_{n-2} F_{n+2}\right)\left(F_{n-1} F_{n+1}-F_{n-2} F_{n+2}\right) \\
& =2 F_{n}^{2}\left(2 F_{n-1} F_{n+1}-2 F_{n}^{2}\right) \\
& =(-1)^{n} \cdot 4 \cdot F_{n}^{2},
\end{aligned}
$$

where the last equality follows from (2.1).

Lemma 3.2 If $n \geq 2$, then

$$
F_{2 n-3} F_{2 n+1}-F_{2 n-1}^{2}=1 \text {. }
$$


Proof It is straightforward to check that

$$
\begin{aligned}
F_{2 n-3} F_{2 n+1}-F_{2 n-1}^{2} & =\left(F_{2 n-1}-F_{2 n-2}\right)\left(F_{2 n-1}+F_{2 n}\right)-F_{2 n-1}^{2} \\
& =F_{2 n-1}^{2}+F_{2 n-1} F_{2 n}-F_{2 n-2} F_{2 n-1}-F_{2 n-2} F_{2 n}-F_{2 n-1}^{2} \\
& =F_{2 n-1} F_{2 n}-F_{2 n-2} F_{2 n-1}-F_{2 n-2} F_{2 n} \\
& =F_{2 n-1} F_{2 n}-F_{2 n-2} F_{2 n+1} \\
& =1,
\end{aligned}
$$

where the last equality follows from (2.6).

Lemma 3.3 For each $n \geq 2$, we have

$$
F_{2 n+1}^{2}-F_{2 n-3}^{2}>3 F_{2 n-1}^{2} \text {. }
$$

Proof A direct calculation shows that

$$
\begin{aligned}
F_{2 n+1}^{2}-F_{2 n-3}^{2} & =\left(F_{2 n+1}+F_{2 n-3}\right)\left(F_{2 n+1}-F_{2 n-3}\right) \\
& =\left(2 F_{2 n-1}+F_{2 n-2}+F_{2 n-3}\right)\left(F_{2 n-1}+F_{2 n}-F_{2 n-3}\right) \\
& >3 F_{2 n-1} F_{2 n-1} \\
& =3 F_{2 n-1}^{2} .
\end{aligned}
$$

The proof is complete.

Remark In fact, applying the equalities (ii) and (iv) of Proposition 2.2 of [17], we can easily obtain

$$
F_{2 n+1}^{2}-F_{2 n-3}^{2}=\left(F_{2 n+1}+F_{2 n-3}\right)\left(F_{2 n+1}-F_{2 n-3}\right)=3 F_{2 n-1} \cdot L_{2 n-1},
$$

where $L_{n}$ means the $n$th Lucas number. Then (3.3) follows immediately from the fact $L_{n}>$ $F_{n}$ for $n \geq 2$.

Now we are ready to present the reciprocal square sums of the Fibonacci numbers.

Theorem 3.4 For all $n \geq 1$ and $m \geq 2$, we have

$$
\left\lfloor\left(\sum_{k=n}^{m n} \frac{1}{F_{2 k}^{2}}\right)^{-1}\right\rfloor=F_{4 n-2}-1 .
$$

Proof It is clearly true for $n=1$, so we assume that $n \geq 2$ in the rest of the proof.

For $k \geq 2$, we have

$$
\begin{aligned}
\frac{1}{F_{4 k-2}-1}-\frac{1}{F_{2 k}^{2}}-\frac{1}{F_{4 k+2}-1} & =\frac{F_{2 k}^{2}\left(F_{4 k+2}-F_{4 k-2}\right)-\left(F_{4 k-2}-1\right)\left(F_{4 k+2}-1\right)}{\left(F_{4 k-2}-1\right) F_{2 k}^{2}\left(F_{4 k+2}-1\right)} \\
& >\frac{F_{2 k}^{2}\left(F_{4 k+2}-F_{4 k-2}\right)-F_{4 k-2} F_{4 k+2}+F_{4 k+2}}{\left(F_{4 k-2}-1\right) F_{2 k}^{2}\left(F_{4 k+2}-1\right)} .
\end{aligned}
$$


It follows from (2.3) that

$$
\begin{aligned}
& F_{2 k}^{2}-F_{2 k-2}^{2}=F_{4 k-2}, \\
& F_{2 k+2}^{2}-F_{2 k}^{2}=F_{4 k+2} .
\end{aligned}
$$

As a consequence of (3.1), we see

$$
F_{2 k-1}^{2} F_{2 k+1}^{2}-F_{2 k-2}^{2} F_{2 k+2}^{2}=4 F_{2 k}^{2}
$$

Applying (2.1), (3.5), (3.6), and (3.7), we derive that

$$
\begin{aligned}
F_{2 k}^{2} & \left(F_{4 k+2}-F_{4 k-2}\right)-F_{4 k-2} F_{4 k+2}+F_{4 k+2} \\
& =F_{2 k}^{2}\left(F_{2 k+2}^{2}-2 F_{2 k}^{2}+F_{2 k-2}^{2}\right)-\left(F_{2 k}^{2}-F_{2 k-2}^{2}\right)\left(F_{2 k+2}^{2}-F_{2 k}^{2}\right)+F_{2 k+2}^{2}-F_{2 k}^{2} \\
& =-F_{2 k}^{4}+F_{2 k-2}^{2} F_{2 k+2}^{2}+F_{2 k+2}^{2}-F_{2 k}^{2} \\
& =-F_{2 k}^{2}\left(F_{2 k}^{2}+1\right)+F_{2 k-2}^{2} F_{2 k+2}^{2}+F_{2 k+2}^{2} \\
& =-\left(F_{2 k-1} F_{2 k+1}-1\right) F_{2 k-1} F_{2 k+1}+F_{2 k-2}^{2} F_{2 k+2}^{2}+F_{2 k+2}^{2} \\
& =-F_{2 k-1}^{2} F_{2 k+1}^{2}+F_{2 k-1} F_{2 k+1}+F_{2 k-2}^{2} F_{2 k+2}^{2}+F_{2 k+2}^{2} \\
& =F_{2 k+2}^{2}-F_{2 k-1}^{2} F_{2 k+1}^{2}+F_{2 k-2}^{2} F_{2 k+2}^{2}+F_{2 k-1} F_{2 k+1} \\
& =F_{2 k+2}^{2}-4 F_{2 k}^{2}+F_{2 k-1} F_{2 k+1} \\
& =\left(F_{2 k+2}-2 F_{2 k}\right)\left(F_{2 k+2}+2 F_{2 k}\right)+F_{2 k-1} F_{2 k+1} \\
& >0
\end{aligned}
$$

which implies that

$$
\frac{1}{F_{4 k-2}-1}-\frac{1}{F_{2 k}^{2}}-\frac{1}{F_{4 k+2}-1}>0 .
$$

Thus, we have

$$
\sum_{k=n}^{m n} \frac{1}{F_{2 k}^{2}}<\frac{1}{F_{4 n-2}-1}-\frac{1}{F_{4 m n+2}-1}<\frac{1}{F_{4 n-2}-1} .
$$

Employing the same argument as above, we obtain, for $k \geq 2$,

$$
\frac{1}{F_{4 k-2}}-\frac{1}{F_{2 k}^{2}}-\frac{1}{F_{4 k+2}}=-\frac{3 F_{2 k}^{2}-F_{2 k-1} F_{2 k+1}}{F_{4 k-2} F_{2 k}^{2} F_{4 k+2}} .
$$

For each $k \geq 2$, we have

$$
\begin{aligned}
3 F_{2 k}^{2}-F_{2 k-1} F_{2 k+1} & =3 F_{2 k}^{2}-F_{2 k-1}\left(F_{2 k-1}+F_{2 k}\right) \\
& =F_{2 k}^{2}+\left(F_{2 k}^{2}-F_{2 k-1}^{2}\right)+\left(F_{2 k}^{2}-F_{2 k-1} F_{2 k}\right) \\
& >F_{2 k}^{2} .
\end{aligned}
$$


Therefore,

$$
\frac{1}{F_{4 k-2}}-\frac{1}{F_{2 k}^{2}}-\frac{1}{F_{4 k+2}}<-\frac{1}{F_{4 k-2} F_{4 k+2}},
$$

from which we arrive at

$$
\begin{aligned}
\sum_{k=n}^{m n} \frac{1}{F_{2 k}^{2}} & >\frac{1}{F_{4 n-2}}-\frac{1}{F_{4 m n+2}}+\frac{1}{F_{4 n-2} F_{4 n+2}} \\
& >\frac{1}{F_{4 n-2}}+\frac{1}{F_{8 n}}-\frac{1}{F_{4 m n+2}} \\
& >\frac{1}{F_{4 n-2}} .
\end{aligned}
$$

Combining (3.8) and (3.9) yields

$$
\frac{1}{F_{4 n-2}}<\sum_{k=n}^{m n} \frac{1}{F_{2 k}^{2}}<\frac{1}{F_{4 n-2}-1},
$$

from which the desired result follows.

As $m$ tends to infinity in Theorem 3.4, we have the following consequence.

Corollary 3.5 For all $n \geq 1$, we have

$$
\left\lfloor\left(\sum_{k=n}^{\infty} \frac{1}{F_{2 k}^{2}}\right)^{-1}\right\rfloor=F_{4 n-2}-1 .
$$

Theorem 3.6 If $n \geq 1$ and $m \geq 2$, then

$$
\left\lfloor\left(\sum_{k=n}^{m n} \frac{1}{F_{2 k-1}^{2}}\right)^{-1}\right\rfloor=F_{4 n-4} .
$$

Proof It is obvious when $n=1$, thus we assume that $n \geq 2$ in the following.

It follows from (2.3) that

$$
\begin{aligned}
& F_{2 k+1}^{2}-F_{2 k-1}^{2}=F_{4 k}, \\
& F_{2 k-1}^{2}-F_{2 k-3}^{2}=F_{4 k-4 .}
\end{aligned}
$$

Therefore, applying (3.2), we deduce

$$
\begin{aligned}
F_{2 k-1}^{2}\left(F_{4 k}-F_{4 k-4}\right)-F_{4 k-4} F_{4 k}= & F_{2 k-1}^{2}\left(F_{2 k+1}^{2}-2 F_{2 k-1}^{2}+F_{2 k-3}^{2}\right) \\
& -\left(F_{2 k+1}^{2}-F_{2 k-1}^{2}\right)\left(F_{2 k-1}^{2}-F_{2 k-3}^{2}\right) \\
= & F_{2 k-3}^{2} F_{2 k+1}^{2}-F_{2 k-1}^{4} \\
= & \left(F_{2 k-3} F_{2 k+1}-F_{2 k-1}^{2}\right)\left(F_{2 k-3} F_{2 k+1}+F_{2 k-1}^{2}\right) \\
= & F_{2 k-3} F_{2 k+1}+F_{2 k-1}^{2} .
\end{aligned}
$$


For $k \geq 2$, we have

$$
\begin{aligned}
\frac{1}{F_{4 k-4}}-\frac{1}{F_{2 k-1}^{2}}-\frac{1}{F_{4 k}} & =\frac{F_{2 k-1}^{2}\left(F_{4 k}-F_{4 k-4}\right)-F_{4 k-4} F_{4 k}}{F_{4 k-4} F_{2 k-1}^{2} F_{4 k}} \\
& =\frac{F_{2 k-3} F_{2 k+1}+F_{2 k-1}^{2}}{F_{4 k-4} F_{2 k-1}^{2} F_{4 k}} \\
& >0
\end{aligned}
$$

from which we derive

$$
\sum_{k=n}^{m n} \frac{1}{F_{2 k-1}^{2}}<\frac{1}{F_{4 n-4}}-\frac{1}{F_{4 m n}}<\frac{1}{F_{4 n-4}} .
$$

Employing (3.2) and (3.12), we obtain

$$
F_{2 k-1}^{2}\left(F_{4 k}-F_{4 k-4}\right)-\left(F_{4 k-4}+1\right)\left(F_{4 k}+1\right)=2 F_{2 k-1}^{2}+F_{2 k-3}^{2}-F_{2 k+1}^{2}<-F_{2 k-1}^{2} \text {, }
$$

where the last inequality follows from (3.3).

Now we see that, for $k \geq 2$,

$$
\begin{aligned}
\frac{1}{F_{4 k-4}+1}-\frac{1}{F_{2 k-1}^{2}}-\frac{1}{F_{4 k}+1} & =\frac{F_{2 k-1}^{2}\left(F_{4 k}-F_{4 k-4}\right)-\left(F_{4 k-4}+1\right)\left(F_{4 k}+1\right)}{\left(F_{4 k-4}+1\right) F_{2 k-1}^{2}\left(F_{4 k}+1\right)} \\
& <\frac{-1}{\left(F_{4 k-4}+1\right)\left(F_{4 k}+1\right)},
\end{aligned}
$$

which implies that

$$
\sum_{k=n}^{m n} \frac{1}{F_{2 k-1}^{2}}>\frac{1}{F_{4 n-4}+1}-\frac{1}{F_{4 m n}+1}+\frac{1}{\left(F_{4 n-4}+1\right)\left(F_{4 n}+1\right)} .
$$

It is easy to see that

$$
\begin{aligned}
\left(F_{4 n-4}+1\right)\left(F_{4 n}+1\right) & =F_{4 n-4} F_{4 n}+F_{4 n-4}+F_{4 n}+1 \\
& <F_{8 n-4}+F_{8 n-3}+F_{8 n-1}+1 \\
& =F_{8 n}+1 .
\end{aligned}
$$

Hence,

$$
\sum_{k=n}^{m n} \frac{1}{F_{2 k-1}^{2}}>\frac{1}{F_{4 n-4}+1}+\frac{1}{F_{8 n}+1}-\frac{1}{F_{4 m n}+1} \geq \frac{1}{F_{4 n-4}+1}
$$

It follows from (3.13) and (3.14) that

$$
\frac{1}{F_{4 n-4}+1}<\sum_{k=n}^{m n} \frac{1}{F_{2 k-1}^{2}}<\frac{1}{F_{4 n-4}}
$$

which completes the proof. 
Consequently, we have the following result.

Corollary 3.7 If $n \geq 1$, then

$$
\left\lfloor\left(\sum_{k=n}^{\infty} \frac{1}{F_{2 k-1}^{2}}\right)^{-1}\right\rfloor=F_{4 n-4} .
$$

\section{Conclusions}

In this paper, we give the exact integral values of the reciprocal sums (resp., square sums) of the even and odd terms in the Fibonacci sequence. The results are new and important for those with closely related research interests. In addition, the methods used here are very elementary and can be extended to the investigation of other combinatorial sequences.

In a future paper, the reciprocal sums and the reciprocal square sums of the Fibonacci 3 -subsequences will be presented.

\section{Competing interests}

The authors declare that they have no competing interests.

\section{Authors' contributions}

All authors contributed equally to deriving all the results of this article, and read and approved the final manuscript.

\section{Acknowledgements}

The authors would like to thank the anonymous referees for their helpful suggestions and comments which improved significantly the presentation of the paper. This work was supported by the National Natural Science Foundation of China (No. 11401080).

Received: 21 August 2015 Accepted: 21 November 2015 Published online: 02 December 2015

\section{References}

1. Duncan, RL: Applications of uniform distribution to the Fibonacci numbers. Fibonacci Q. 5, 137-140 (1967)

2. Holliday, SH, Komatsu, T: On the sum of reciprocal generalized Fibonacci numbers. Integers 11A, Article 11 (2011)

3. Karaduman, E: An application of Fibonacci numbers in matrices. Appl. Math. Comput. 147, $903-908$ (2004)

4. Koshy, T: Fibonacci and Lucas Numbers with Applications. Wiley, New York (2001)

5. Vorobiev, NN: Fibonacci Numbers. Birkhäuser, Basel (2002)

6. Elsner, C, Shimomura, S, Shiokawa, I: Algebraic relations for reciprocal sums of Fibonacci numbers. Acta Arith. 130, 37-60 (2007)

7. Elsner, C, Shimomura, S, Shiokawa, I: Algebraic relations for reciprocal sums of odd terms in Fibonacci numbers. Ramanujan J. 17, 429-446 (2008)

8. Elsner, C, Shimomura, S, Shiokawa, I: Algebraic independence results for reciprocal sums of Fibonacci numbers. Acta Arith. 148, 205-223 (2011)

9. Elsner, C, Shimomura, S, Shiokawa, I: Algebraic relations for reciprocal sums of even terms in Fibonacci numbers. J. Math. Sci. 180, 650-671 (2012)

10. Ohtsuka, H, Nakamura, S: On the sum of reciprocal Fibonacci numbers. Fibonacci Q. 46/47, 153-159 (2008/2009)

11. Wu, ZG, Zhang, WP: The sums of the reciprocals of Fibonacci polynomials and Lucas polynomials. J. Inequal. Appl. 2012, Article ID 134 (2012)

12. Wu, ZG, Zhang, WP: Several identities involving the Fibonacci polynomials and Lucas polynomials. J. Inequal. Appl. 2013, Article ID 205 (2013)

13. Wu, ZG, Wang, TT: The finite sum of reciprocal of the Fibonacci numbers. J. Inn. Mong. Norm. Univ., Nat. Sci. 40, 126-129 (2011)

14. Wang, AYZ, Wen, PB: On the partial finite sums of the reciprocals of the Fibonacci numbers. J. Inequal. Appl. 2015, Article ID 73 (2015)

15. Akyiğit, M, Kösal, HH, Tosun, M: Split Fibonacci quaternions. Adv. Appl. Clifford Algebras 23, 535-545 (2013)

16. Akyiğit, M, Kösal, HH, Tosun, M: Fibonacci generalized quaternions. Adv. Appl. Clifford Algebras 24, 631-641 (2014)

17. Savin, D: Some properties of Fibonacci numbers, Fibonacci octonions, and generalized Fibonacci-Lucas octonions. Adv. Differ. Equ. 2015, Article ID 298 (2015) 